\section{nephron \\ Clinical}

Practice
Nephron 2017;136:268-272

DOI: $10.1159 / 000456621$
Received: September 1, 2016

Accepted after revision: December 13, 2016

Published online: February 18, 2017

\title{
Drug Dosing and Estimated Renal Function - Any Step Forward from Effersoe?
}

\author{
Mads Hornum Bo Feldt-Rasmussen \\ Department of Nephrology, Copenhagen University Hospital, Rigshospitalet, Copenhagen, Denmark
}

\section{Keywords}

Glomerular filtration rate estimation - Drug dosing .

Equation performance

\section{Abstract}

Drug dosing in accordance with the renal function is a longstanding challenge to clinicians. For many years it has been evident that in many clinical situations there is no easy way to correctly dose any drug that is mainly cleared by the kidneys. Despite the development of many formulas for estimating the glomerular filtration rate, they all have serious shortcomings. Much effort has been put in to develop estimation formulas to evaluate the renal function as an alternative to direct methods with the aim of safely dosing drugs that are mainly cleared by the kidneys. Both creatinine- and cystatin C-based formulas with additional clinical and biochemical parameters deduced from association studies with methods to measure the glomerular filtration rate (mGFR) have been developed. None of them have been good enough to perform safely in the wide range of situations in daily clinical praxis. Despite serious limitations, there has also been a tendency to use estimated GFR (eGFR) as a "hard" clinical endpoint in clinical studies. This has increased the risk of misinterpretation and has led to conclusions that are not necessarily supported by data. Finally, new methods of

\section{KARGER}

(C) 2017 S. Karger AG, Basel

E-Mail karger@karger.com

www.karger.com/nef testing drug toxicity and the use of pharmacological support in order to fix the right doses are mentioned in this short overview of studies; possible problems that are encountered using eGFR instead of mGFR in the clinic and in research are also mentioned in this report.

(c) 2017 S. Karger AG, Basel

\section{Introduction}

In 1956, doctor Effersoe [1] from Copenhagen, Denmark, published a paper about the relationship between endogenous $24 \mathrm{~h}$ creatinine clearance against serum creatinine. Inulin clearance was the gold standard at that time and there were much debate about the clinical validity of creatinine clearance, especially in terms of its low values. It was done in patients with chronic nephropathy of unknown etiology, in "steady state." $\mathrm{He}$ developed a nomogram for determining the percent renal function, given a specific serum creatinine value for use in dosing of drugs among other things. An example for its use was adjusting the dose of digoxin according

Contribution from the CME course of the DIABESITY Working Group of the ERA-EDTA, Bergamo, December 4-5, 2015.

Prof. Bo Feldt-Rasmussen 
to the renal function using this simple nomogram. Introducing this estimated serum creatinine-based estimated GFR (eGFR) was a giant step because it was easy to use and increased the focus on the need for correcting dosages of many drugs that were mainly cleared by the kidneys. On the other hand, this method over- or underestimated the renal function in so many cases, since it was dependent on many factors such as age, gender, race, body mass, muscle mass, food intake and a stable state of hydration and clinical situation in general. So, the method added important new information for use in safe drug-dosing, but it was not perfect even though it was closely related to a renal clearance method using an endogenous marker of kidney function (creatinine). Since then, numerous new serum (S)-creatinine-based formulas of eGFR have taken over, but we postulate that no major changes have been seen in the last 60 years, and that we still have problems with the use of estimating methods for drug dosing. In the perfect world, the eGFR always reflects the actual GFR and therefore would be a reliable/safe base for calculating a loading dose and the continuous dosing of any drug in any clinical situation, cleared by the kidney. Very often it is not, and this is discussed in the following section.

Access to an instant and exact estimate of GFR is mandatory to all clinicians treating severely ill patients and to all clinicians dosing life-saving drugs in cancer treatment and in so many other situations. Such methods are, however, rarely used (i.e., methods using more specific glomerular filtration markers). Many find the methods expensive and cumbersome. Therefore, and instead of doing the right thing, many creatinine-based methods have been developed in an attempt to improve the situation. These include algorithms based on S-creatinine and few clinical parameters with or without urine collections: creatinine clearance, Cockcroft Gault (CG), Chronic Kidney Disease Epidemiology Collaboration (CKD-EPI), Modification of Diet in Renal Disease (MDRD) [2], or algorithms based on $\mathrm{P}$-creatinine in combination with $\mathrm{P}$-cystatin C or P-cystatin C alone [3]. Approaches to get closer to using a golden standard methodology such as the most accurate methods using constant infusion techniques with urine collections, infusing specific filtration markers such as inulin, ${ }^{51}$ Cr-EDTA [4], iohexol [5], iothalamate [6] or others are rarely used even in clinical research. We believe that this is inexpedient. These markers can all be used in much simpler settings using bolus injection, timed plasma sampling (perhaps only one), thereby obtaining quicker and much more reliable estimates of GFR.

Drug Dosing and eGFR

\section{Limitations of Estimated Creatinine Clearance and Urinary Creatinine Clearance Methods}

The eGFR methods are based on correlations or linear regression models between $\mathrm{S}$-creatinine, $\mathrm{P}$-cystatin $\mathrm{C}$ or both and variable patient variables and are also based on measuring the glomerular filtration rate (mGFR), in rather heterogeneous populations. Despite high correlation quotients between eGFR and mGFR seen in many of these studies, it is questionable whether such correlations reflect a clinically useful agreement between eGFR and mGFR in different patient populations, which most often they do not $[7,8]$.

The creatinine clearance method overestimates clearance due to the secretion of creatinine in tubules [9] and is not appropriate for patients with ascites and oedemas, in elderly patients with low muscle mass, or in young patients with large muscle mass or on special diets.

mGFR with an exogenous filtration marker is the most accurate, either with inulin, ${ }^{51} \mathrm{Cr}$-EDTA-clearance, ${ }^{99 \mathrm{~m}}$ Tc-DTPA, iohexol or iothalamate [10], and in the clinic especially, the ${ }^{51} \mathrm{Cr}$-EDTA clearance [4] and iohexol [11] methods are rather simple and cost effective to use, and have in many studies shown excellent performance and precision [10].

\section{Estimated Glomerular Clearance Equations versus Direct Glomerular Filtration Measurements as Endpoints in Clinical Studies}

There are many controversies in the estimation methods used in the field of drug monitoring. This practice is, however, widely used and finds support in reviews [12]; FDA actually recommends that endpoints, safety and dosing of new drugs are being validated on the basis of estimating methods [13]. We are not claiming that there are no arguments at all for using methods estimating GFR, but we do have our anxieties in this regard as argued below where we discuss drug dosing in cancer patients. Among those in favour of using eGFR, drugs were, after Effersoe, historically dosed according to the CG [14] equation with all its weaknesses. The development of better methods like the MDRD [15] equation also gave rise to problems with interpretation of drug recommendations in all but relatively healthy and stable patient populations. Using the formula does however find support. Thus, a large study by Stevens et al. [12] compared the concordance of assigned kidney function categories designated by the FDA [13] with CG and MDRD equations

Nephron 2017;136:268-272

DOI: $10.1159 / 000456621$ 


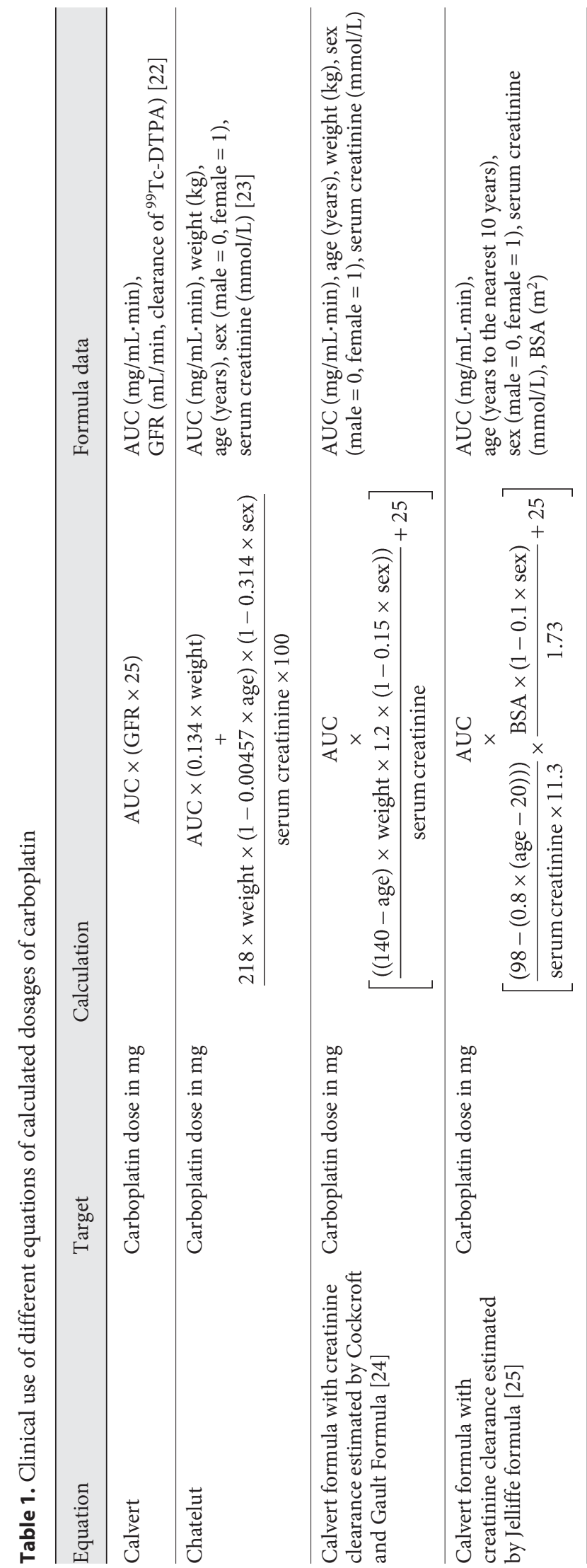

compared with iothalamate excretion in the urine. There were a rather good concordance with the MDRD equation compared to the CG equation in terms of iothalamate clearance, and this was tested for 15 recommended drug doses of medications cleared by the kidneys. In conclusion, they recommended that the MDRD should be used for drug dosing instead of CG until better equations were developed.

This may be true for less toxic drugs with a wide therapeutic window. However, too many errors are involved in the use of eGFR-creatinine- or cystatin C-based formulas to dose drugs, to unstable, often malnourished sarcopenic critically ill patients in the hospital wards. We exemplify this in the next section describing problems in drug dosing after guidance of eGFR in patients treated with cisplatin.

\section{Drug Dosing in Cancer Patients}

The use of eGFR methods have only sparsely been investigated in cancer patients [16]. Caution should especially be exercised when estimation methods are used in dosing of drugs affecting tubular function and creatinine secretion, giving rise to an underestimation of renal function and a risk of too low dosage of a lifesaving drug.

Calvert et al. [22] developed a simple formula for carboplatin dosing and the equation was developed with a gold standard to measure GFR; later it was adjusted with CG eGFR, giving rise to the same problems with sampling errors and overestimation as traditional eGFR methods (Table 1). Dooley et al. [17] demonstrated that the Chatelut formula overestimated the carboplatin dose, especially in men and a gender difference was present in the different formulas. Furthermore, many of the equations used to calculate carboplatin dose used older assays to determine serum creatinine, which was another source of error [18].

Our group participated in a study where cisplatin was used for the chemotherapy (CT) of male germ cell cancer patients. The cohort was without major comorbidities and had good survival benefits. We also studied the eGFR formula's (by MDRD, Wright, CKD-EPI, and CG) [14, $15,19,26]$ influence on hypothetical carboplatin dosage, a drug much used in cancer treatment.

The level of agreement in hypothetically derived carboplatin dosages was calculated. The values after treatment were divided into cisplatin dose strata equivalent to the number of treatment cycles. MDRD offered the best bias, precision and accuracy after CT, 3 and 4 cycles. The 
other equations were more imprecise. In patients receiving more than 5 cycles of CT, more than $62 \%$ of the patients had an eGFR that differed from mGFR by more than $30 \%$. If the equations were used to calculate carboplatin dosage, the doses given would generally be too high, especially when the CKD-EPI and CG equations were used.

Post-CT mGFR decreased in comparison to pretreatment $\mathrm{mGFR}$, a median of $9 \mathrm{~mL} / \mathrm{min}$ after 3 cycles, $14 \mathrm{~mL} /$ min after 4 cycles and $20 \mathrm{~mL} / \mathrm{min}$ after +5 cycles. All eGFR estimates, by the MDRD, Wright and CKD-EPI equations, except for the CG equation, increased significantly after CT. During follow-up at 1, 3, and 5 years, all estimates decreased and mGFR increased significantly [27].

\section{New Methods for Drug Dosing}

There are some hospitals where they do even better having established pharmacological or biochemistry departments that help in calculating the subsequent dosing (amount and interval) on the basis of measured plasma levels of the drug in question, typically an antibiotic. It does not appear that kidney function estimates are needed for that approach; what they are essentially doing is using the antibiotic as the filtration marker and calculating its clearance.

New methods are emerging, like kidney-on-a-chip $[20,21]$ where renal tubular cells have been integrated with microfluidic devices and used to measure the toxicity of an antibiotic. This has large perspectives if it is de- veloped to act as a fast and bedside method, but still a lot of validation studies are needed before it can replace, for example, the measurement of drug concentration.

There is a potential problem with the fact that drug clearance in many of these chips are based on animal cells; drug clearance is higher in animals than in humans, giving rise to a bias in the judgement of toxicity, and human cell lines should be introduced and validated.

\section{Conclusions}

Using eGFR uncritically and as a new golden standard in planning drug dosing is often wrong and unsafe. All the pitfalls and biases behind a correct, accurate and precise eGFR must always be kept in mind and in many cases more direct methods of GFR should be considered in daily clinical practice.

\section{Acknowledgements}

Both authors participated equally in performance of the mini review, data analysis and writing of the paper.

\section{Funding Sources}

M.H. was supported by the Helen Bjørnow Foundation.

\section{Disclosure Statement}

The authors have no conflicts to declare.

\section{References}

1 Effersoe P: Relationship between endogenous 24-hour creatinine clearance and serum creatinine concentration in patients with chronic renal disease. Acta Med Scand 1957;156:429-434.

2 Moellering RC Jr, Krogstad DJ, Greenblatt DJ: Vancomycin therapy in patients with impaired renal function: a nomogram for dosage. Ann Intern Med 1981;94:343-346.

3 Frazee EN, Rule AD, Herrmann SM, Kashani KB, Leung N, Virk A, et al: Serum cystatin C predicts vancomycin trough levels better than serum creatinine in hospitalized patients: a cohort study. Crit Care 2014;18:R110.

4 Brochner-Mortensen J: A simple method for the determination of glomerular filtration rate. Scand J Clin Lab Invest 1972;30:271-274.

5 Krutzen E, Back SE, Nilsson-Ehle I, NilssonEhle P: Plasma clearance of a new contrast agent, iohexol: a method for the assessment of glomerular filtration rate. J Lab Clin Med 1984;104:955-961.

6 Isaka Y, Fujiwara Y, Yamamoto S, Ochi S, Shin S, Inoue T, et al: Modified plasma clearance technique using nonradioactive iothalamate for measuring GFR. Kidney Int 1992;42: 1006-1011.

7 Froissart M, Rossert J, Jacquot C, Paillard M, Houillier P: Predictive performance of the modification of diet in renal disease and Cockcroft-Gault equations for estimating renal function. J Am Soc Nephrol 2005; 16:763773.

8 Stevens LA, Coresh J, Schmid CH, Feldman HI, Froissart M, Kusek J, et al: Estimating GFR using serum cystatin $\mathrm{C}$ alone and in combination with serum creatinine: a pooled analysis of 3,418 individuals with CKD. Am J Kidney Dis 2008;51:395-406.
9 Rapoport A, Husdan H: Endogenous creatinine clearance and serum creatinine in the clinical assessment of kidney function. Can Med Assoc J 1968;99:149-156.

10 Stevens LA, Coresh J, Greene T, Levey AS: Assessing kidney function - measured and estimated glomerular filtration rate. $\mathrm{N}$ Engl J Med 2006;354:2473-2483.

11 Gaspari F, Perico N, Ruggenenti P, Mosconi L, Amuchastegui CS, Guerini E, et al: Plasma clearance of nonradioactive iohexol as a measure of glomerular filtration rate. J Am Soc Nephrol 1995;6:257-263.

12 Stevens LA, Nolin TD, Richardson MM, Feldman HI, Lewis JB, Rodby R, et al: Comparison of drug dosing recommendations based on measured GFR and kidney function estimating equations. Am J Kidney Dis 2009;54:3342 . 
13 Food and Drug Administration: Guidance for Industry: Pharmacokinetics in Patients with Impaired Renal Function Study Design, Data Analysis, and Impact on Dosing and Labeling. Rockville, US Department of Health and Human Services, 1998.

14 Cockcroft DW, Gault MH: Prediction of creatinine clearance from serum creatinine. Nephron 1976;16:31-41.

15 Levey AS, Bosch JP, Lewis JB, Greene T, Rogers N, Roth D: A more accurate method to estimate glomerular filtration rate from serum creatinine: a new prediction equation. Modification of Diet in Renal Disease Study Group. Ann Intern Med 1999;130: 461-470.

16 Hartlev LB, Boeje CR, Bluhme H, Palshof T, Rehling M: Monitoring renal function during chemotherapy. Eur J Nucl Med Mol Imaging 2012;39:1478-1482.
17 Dooley MJ, Poole SG, Rischin D, Webster LK: Carboplatin dosing: gender bias and inaccurate estimates of glomerular filtration rate. Eur J Cancer 2002;38:44-51.

18 Collins IM, Roberts-Thomson R, Faulkner D, Rischin D, Friedlander M, Mileshkin L: Carboplatin dosing in ovarian cancer: problems and pitfalls. Int J Gynecol Cancer 2011;21: 1213-1218.

19 Levey AS, Stevens LA, Schmid CH, Zhang YL, Castro AF 3rd, Feldman HI, et al: A new equation to estimate glomerular filtration rate. Ann Intern Med 2009;150:604-612.

20 Kim S, Takayama S: Organ-on-a-chip and the kidney. Kidney Res Clin Pract 2015;34:165169.

21 Kim S, LesherPerez SC, Kim BC, Yamanishi C, Labuz JM, Leung B, et al: Pharmacokinetic profile that reduces nephrotoxicity of gentamicin in a perfused kidney-on-a-chip. Biofabrication 2016;8:015021.

22 Calvert AH, Newell DR, Gumbrell LA, O’Reilly S, Burnell M, Boxall FE, et al: Carboplatin dosage: prospective evaluation of a simple formula based on renal function. J Clin Oncol 1989;7:1748-1756.
23 Chatelut E, Canal P, Brunner V, Chevreau C, Pujol A, Boneu A, et al: Prediction of carboplatin clearance from standard morphological and biological patient characteristics. J Natl Cancer Inst 1995;87:573-580.

24 Cockcroft DW, Gault MH: Prediction of creatinine clearance from serum creatinine. Nephron 1976;16:31-41.

25 Jelliffe RW: Letter: creatinine clearance: bedside estimate. Ann Intern Med 1973;79:604605.

26 Wright JG, Boddy AV, Highley M, Fenwick J, McGill A, Calvert AH: Estimation of glomerular filtration rate in cancer patients. Br J Cancer 2001;84:452-459.

27 Lauritsen J, Gundgaard MG, Mortensen MS, Oturai PS, Feldt-Rasmussen B, Daugaard G: Reliability of estimated glomerular filtration rate in patients treated with platinum containing therapy. Int J Cancer 2014;135:17331739 . 